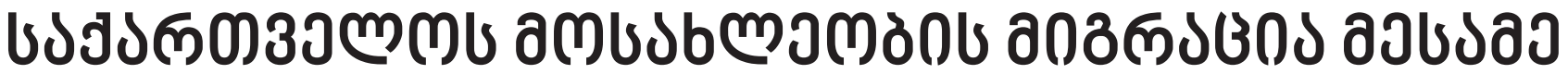

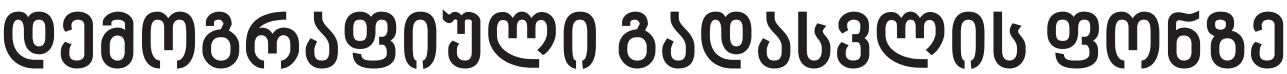

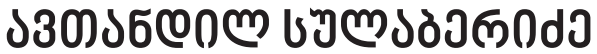

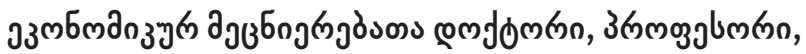

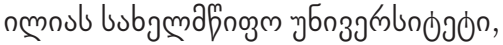

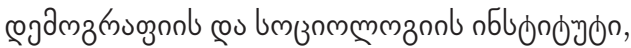

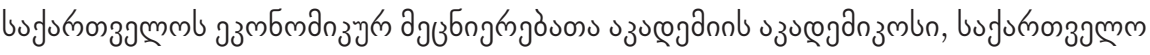

sulaberidzeavtandil@gmail.com

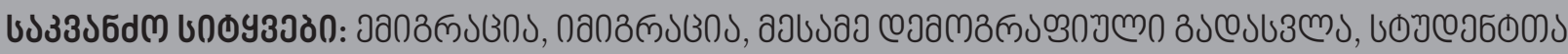

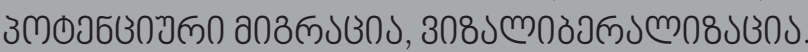

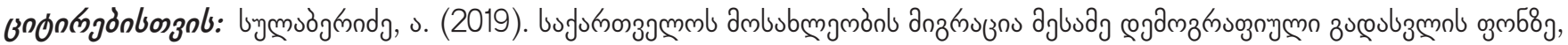

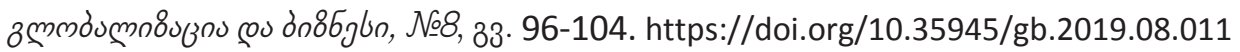

\section{วอง১3১ल00}

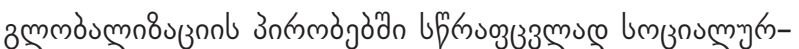

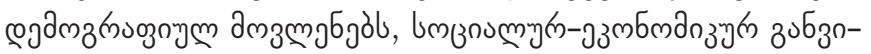

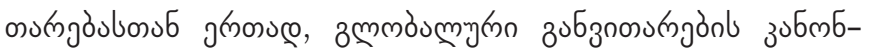

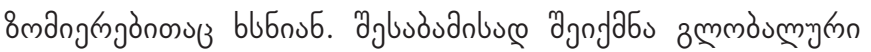

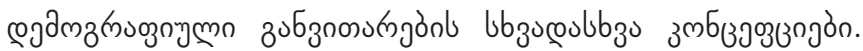

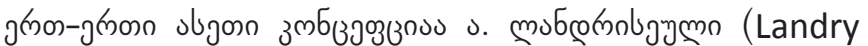

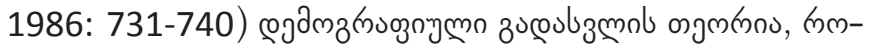

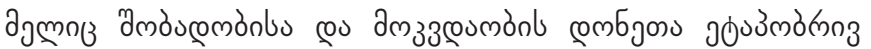

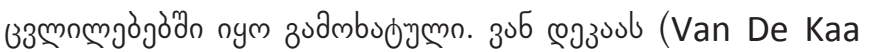
1976:45) Әпgर

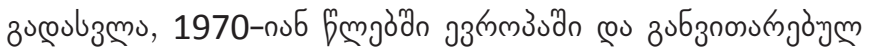

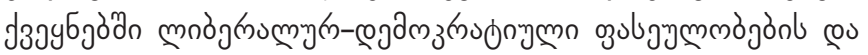

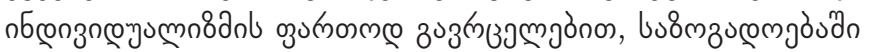

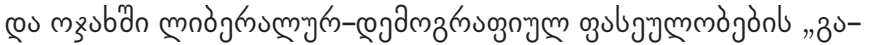

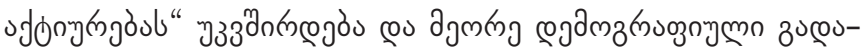

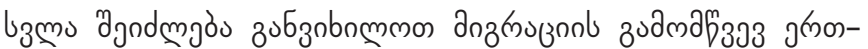

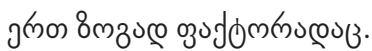

\section{*** $*$}

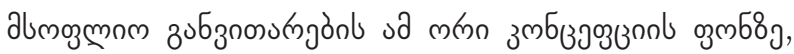

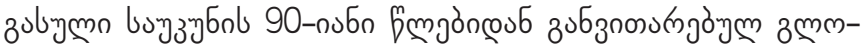

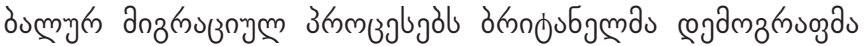

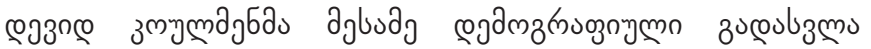

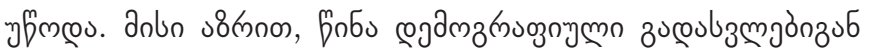

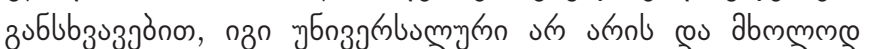

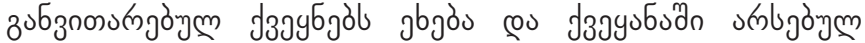

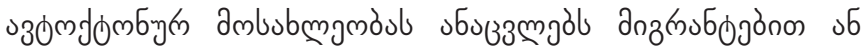

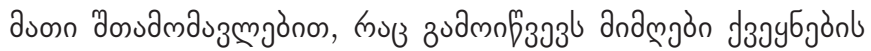

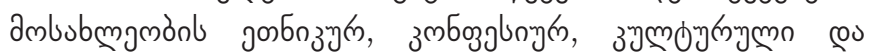

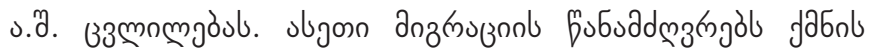

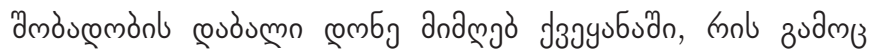

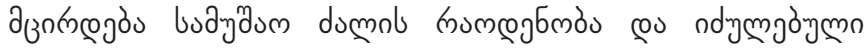

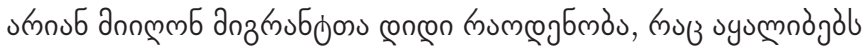

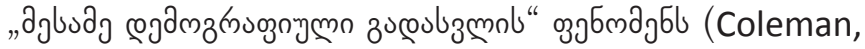
2004).

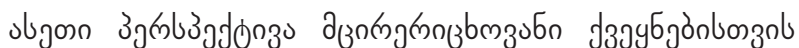

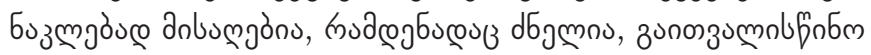

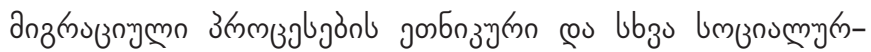

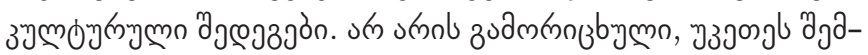

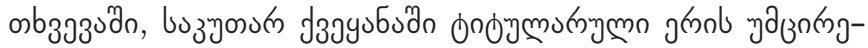

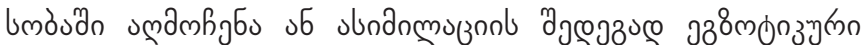

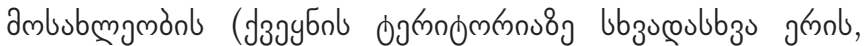

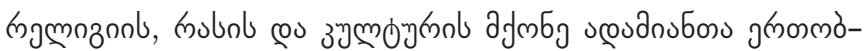

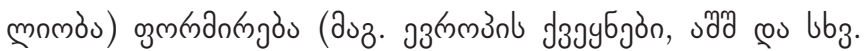

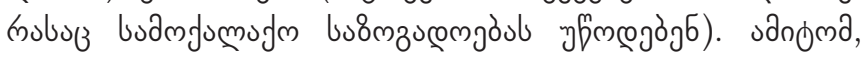

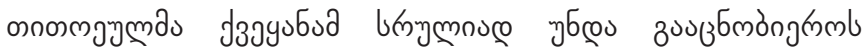

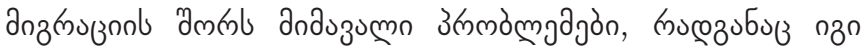

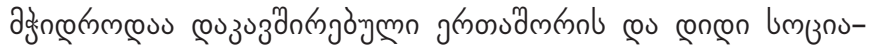

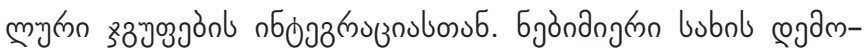

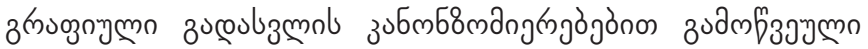

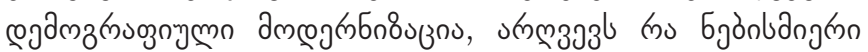

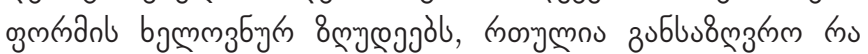




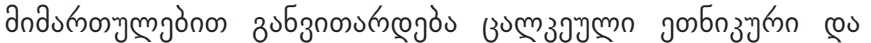

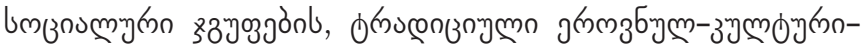

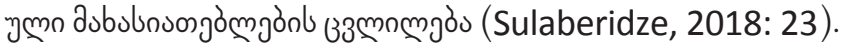

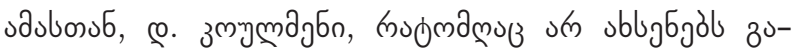

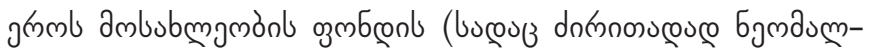

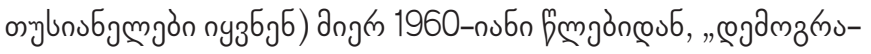

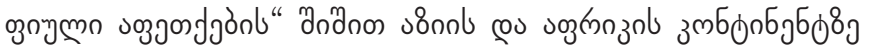

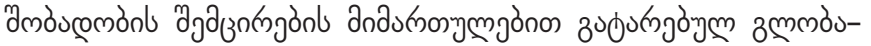

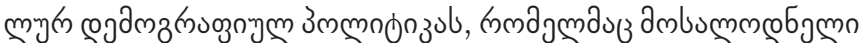

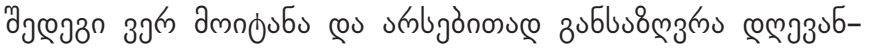

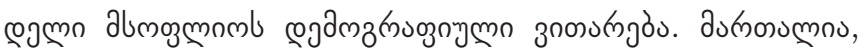

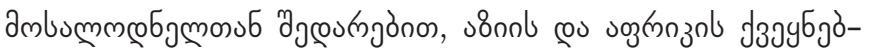

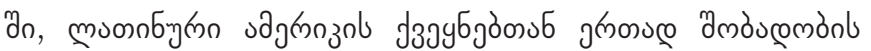

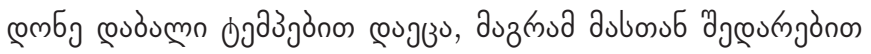

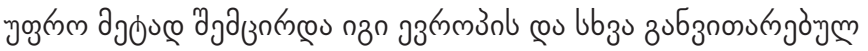
ذзэyб

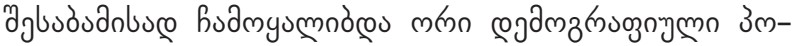

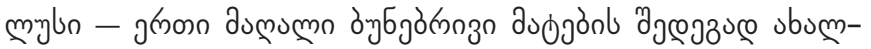

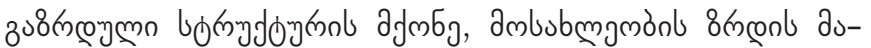

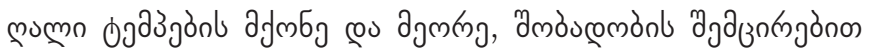

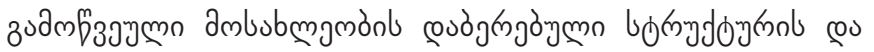

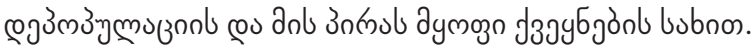

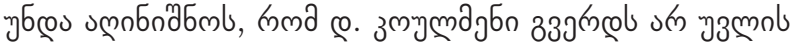

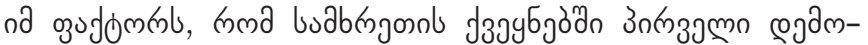

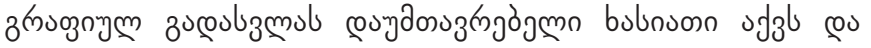

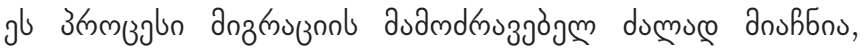

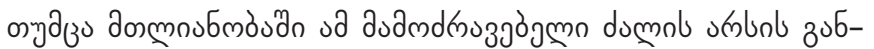

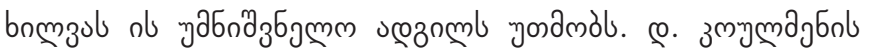

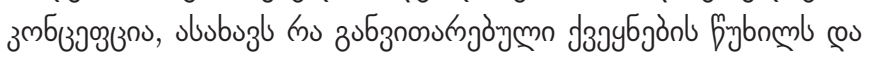

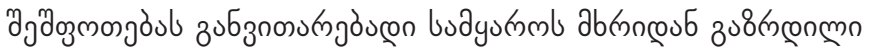

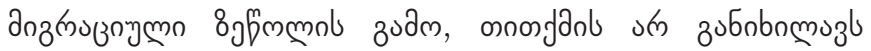

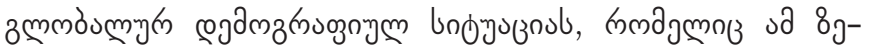

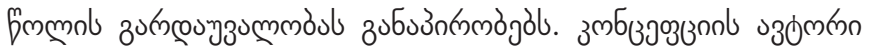

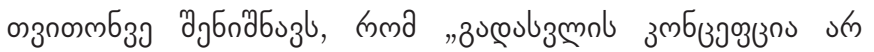

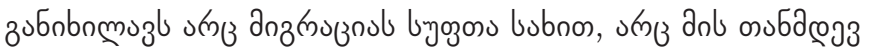

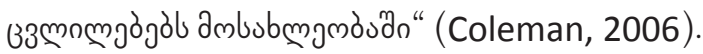

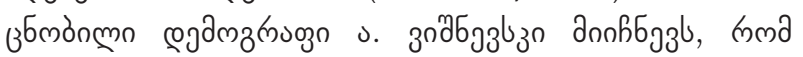

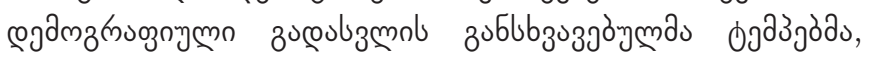

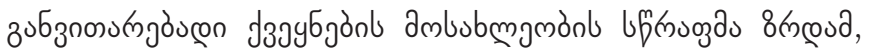

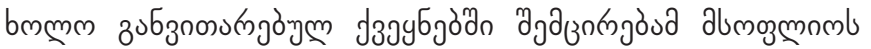

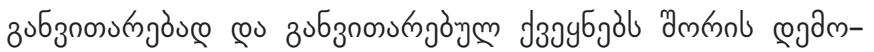

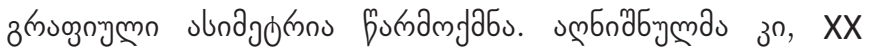

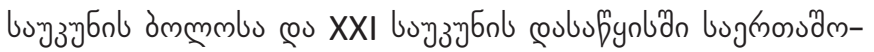

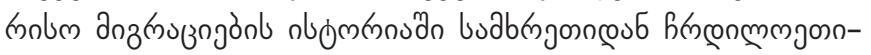

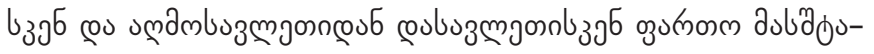

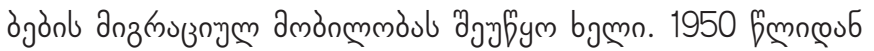

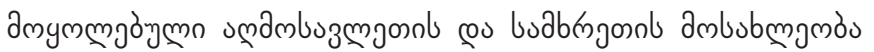

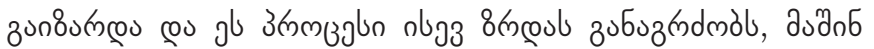

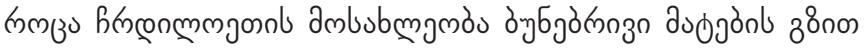

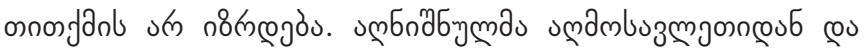

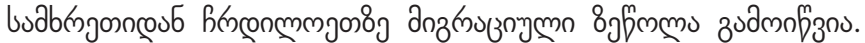

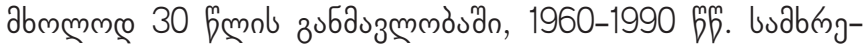

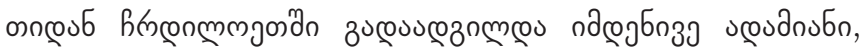

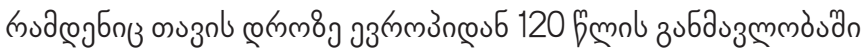

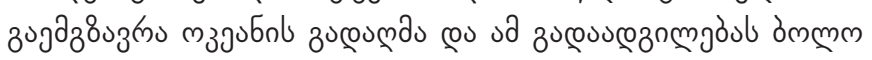

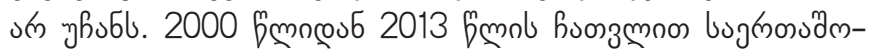
molum anzinubjons mnozbas 57 anmombnoub 232 anmombn gju@̧nбı (Vishnevski, 2015:31).

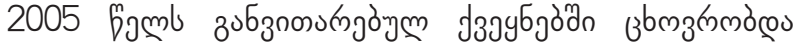

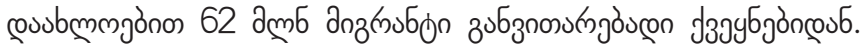

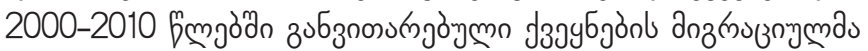

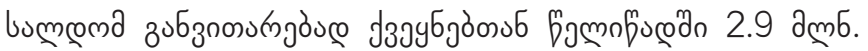

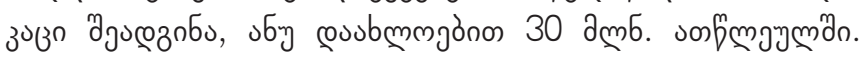

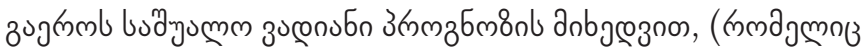

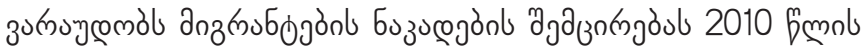

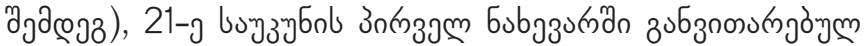

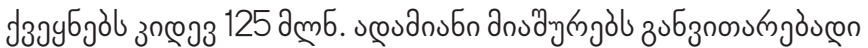
buayummoub (International Migration. 2013).

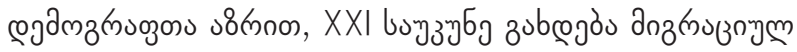
उu@

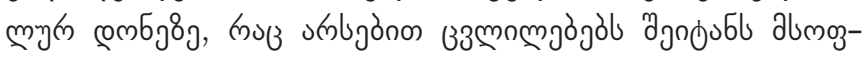

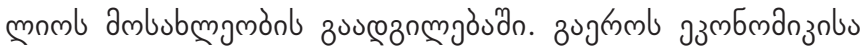

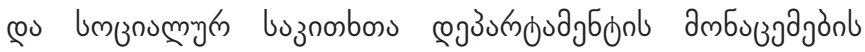
mububdue, 2017 fmolonzol abmogmoman 258 anmombn

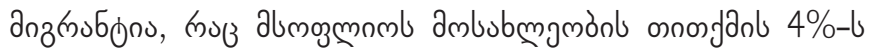

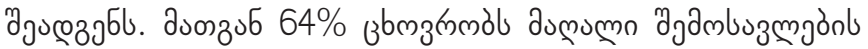

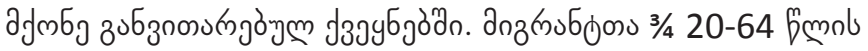

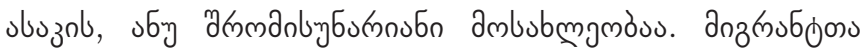

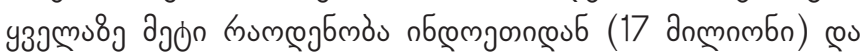

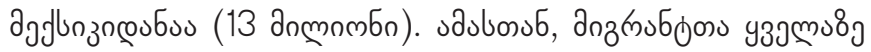

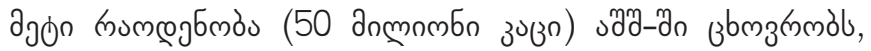

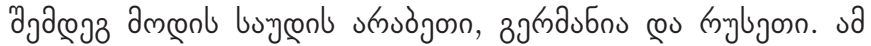

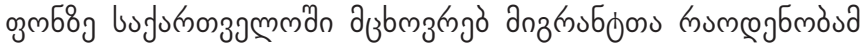

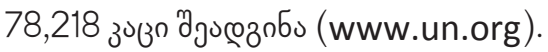

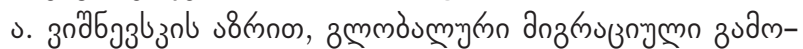

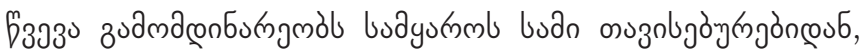

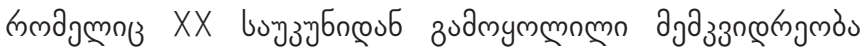

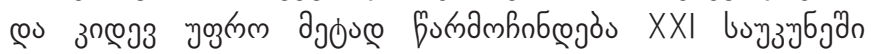

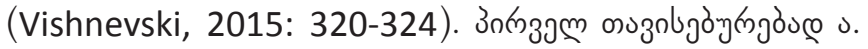
zодб

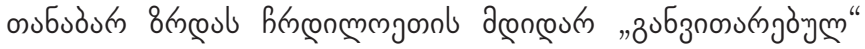

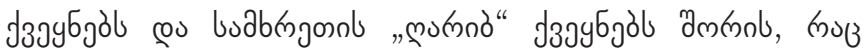

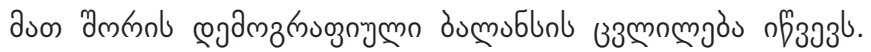

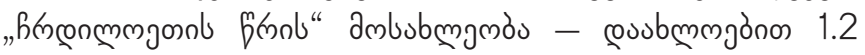

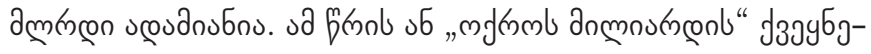

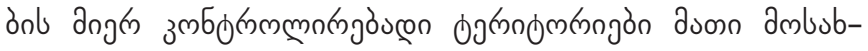

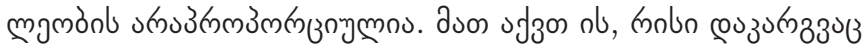

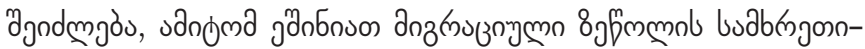

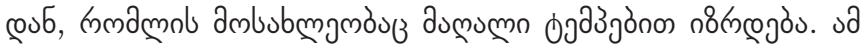

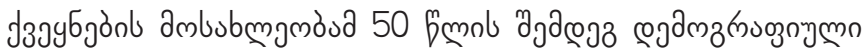




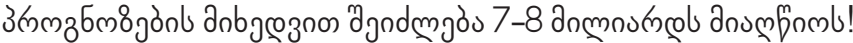

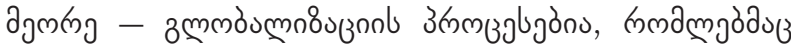

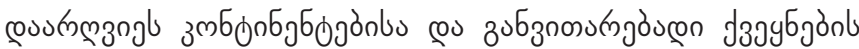

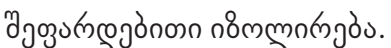

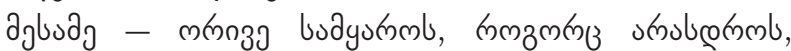

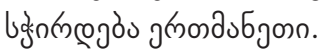

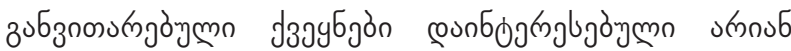

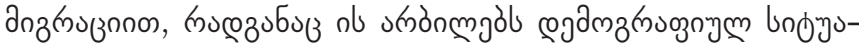

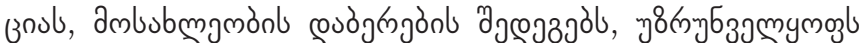

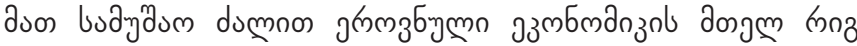

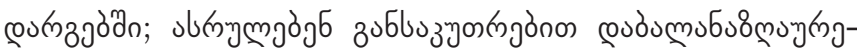

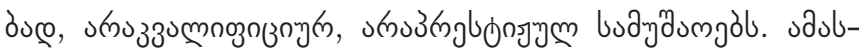

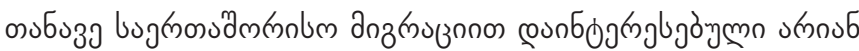

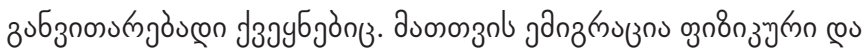

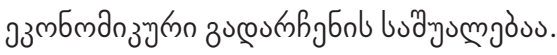

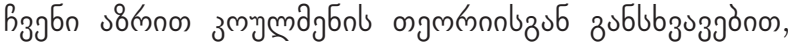

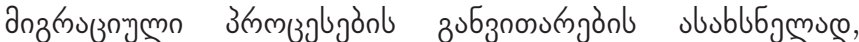

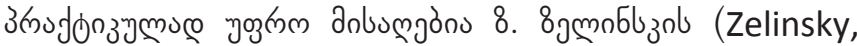

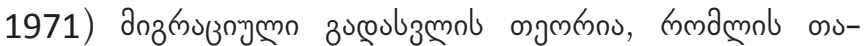

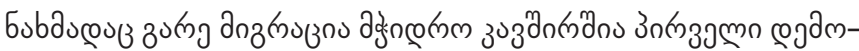

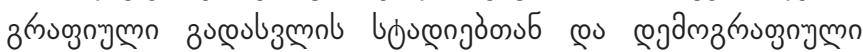

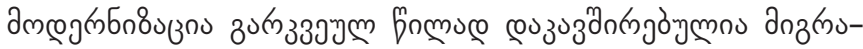

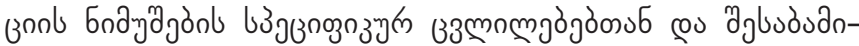

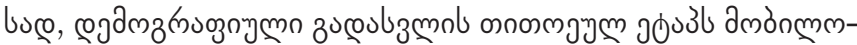

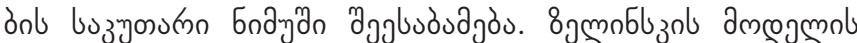

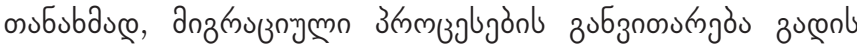

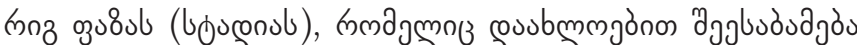

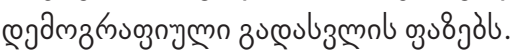

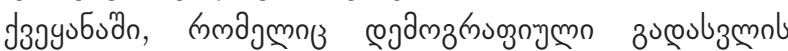

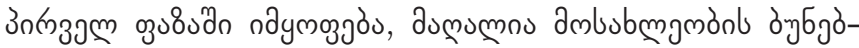

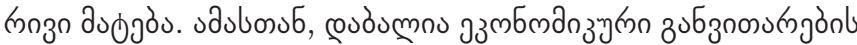

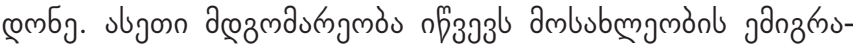

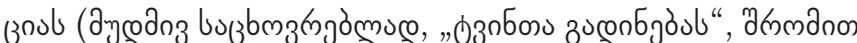

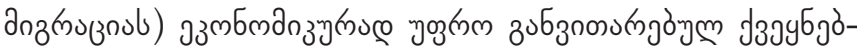

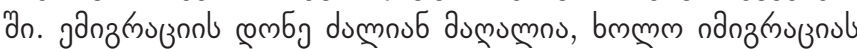

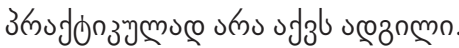

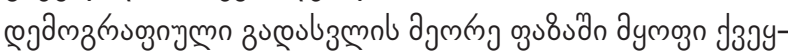

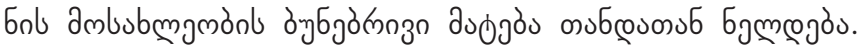

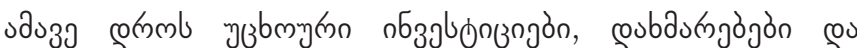

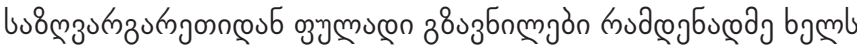

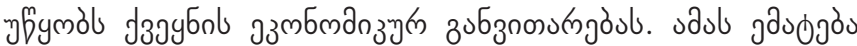

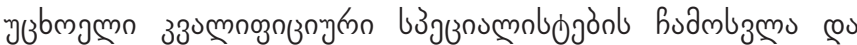

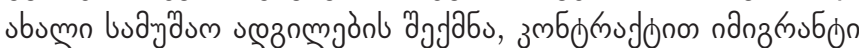

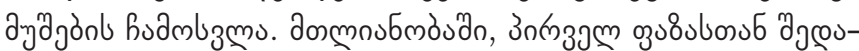

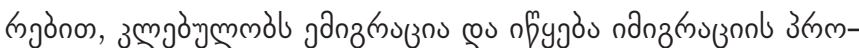

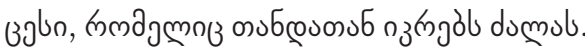

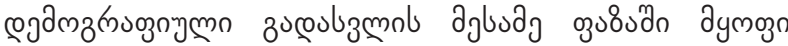

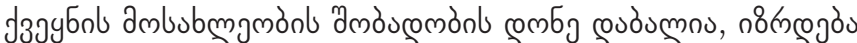

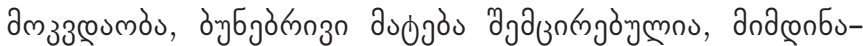

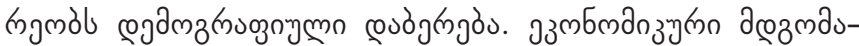

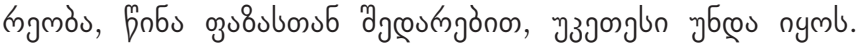

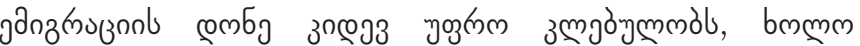

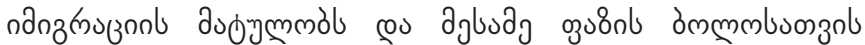

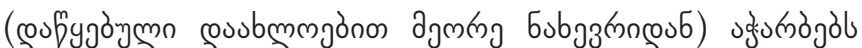

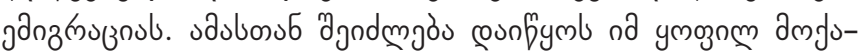

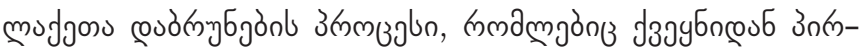

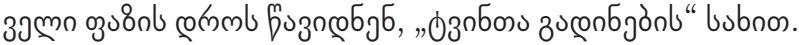

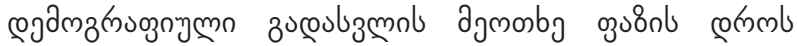

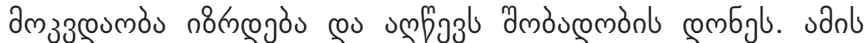

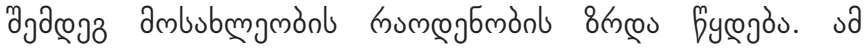

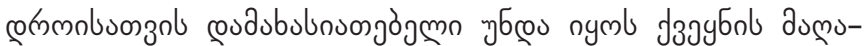

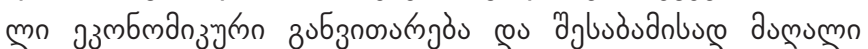

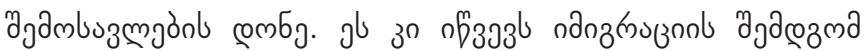

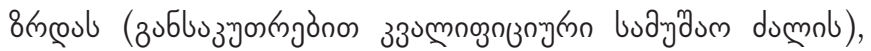

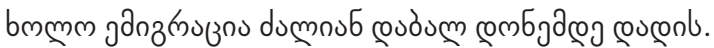

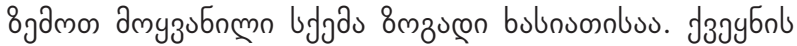

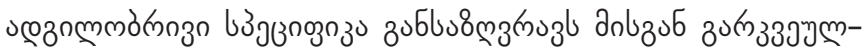

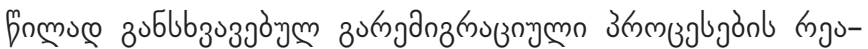

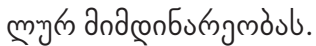

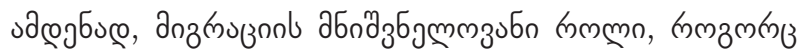

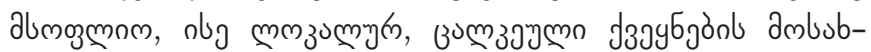

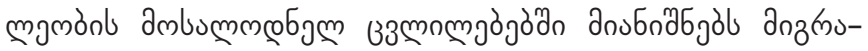

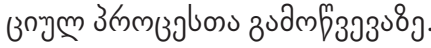

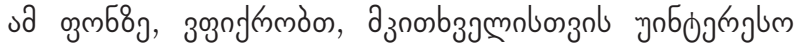

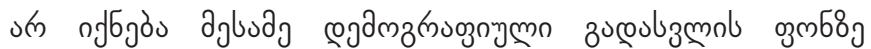

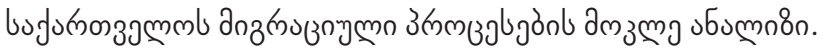

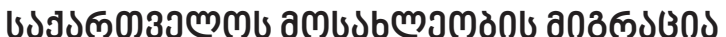

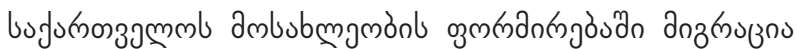

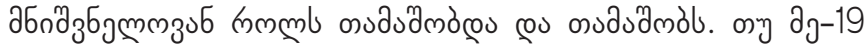

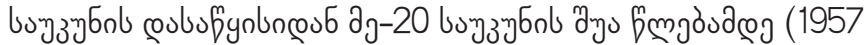

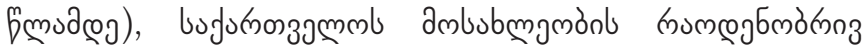

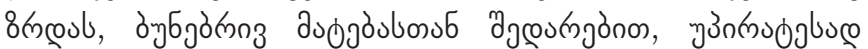

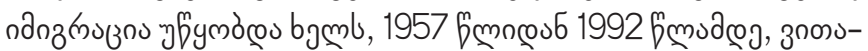

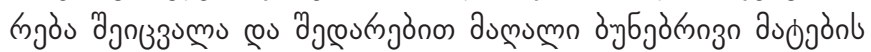

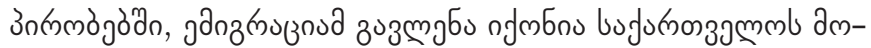

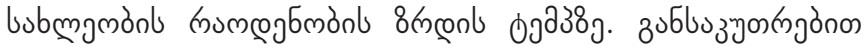

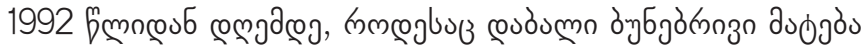

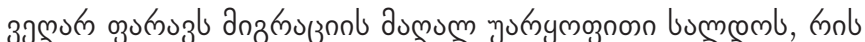

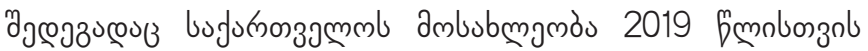

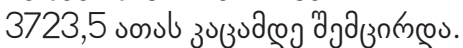

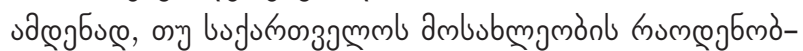

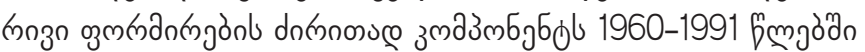

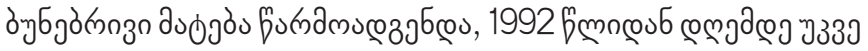

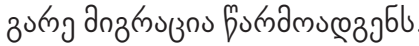

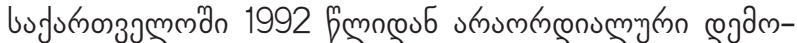

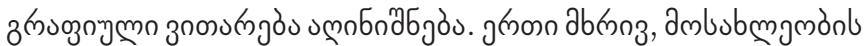

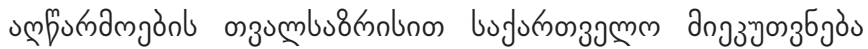




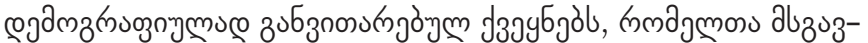

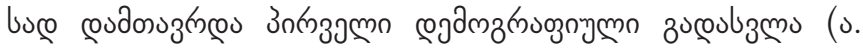

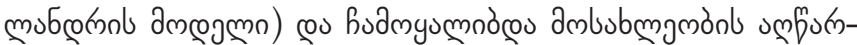

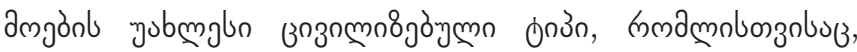

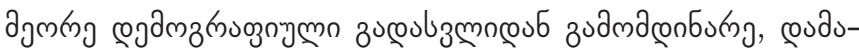

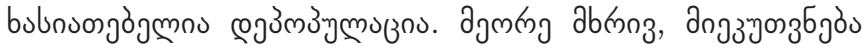

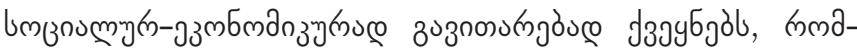

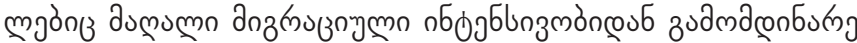

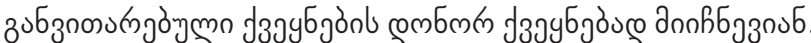

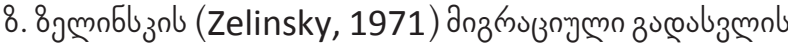

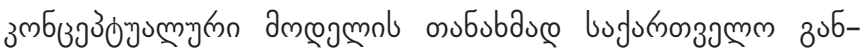

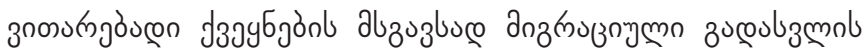

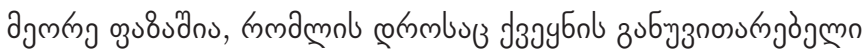

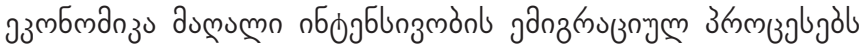

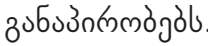

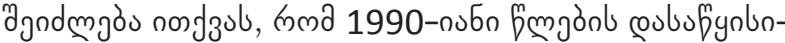

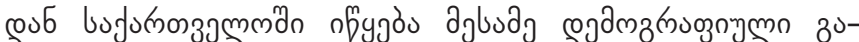

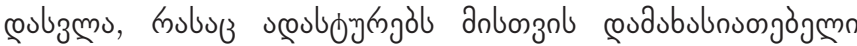

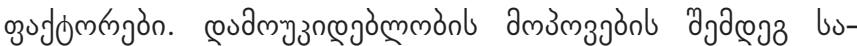

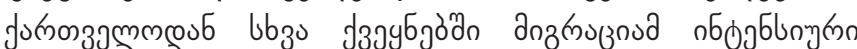

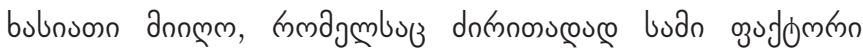

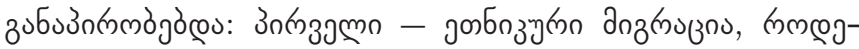

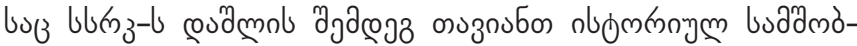

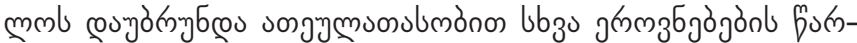

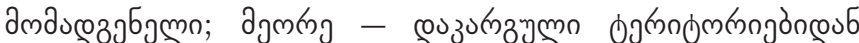

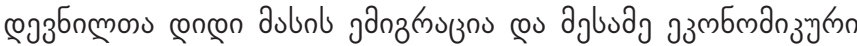
zmmuoglo (Sulaberidze, 2019; Hakkert, 2017:60-69).

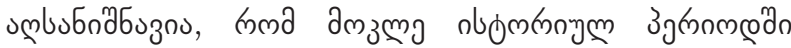

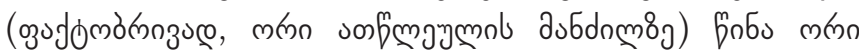

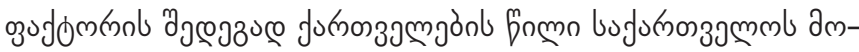

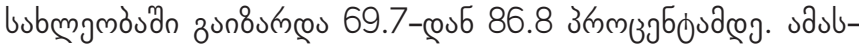

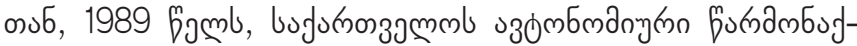
abjonl - sogbusgonol sзб

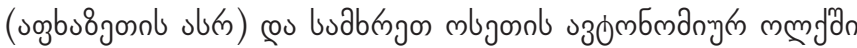

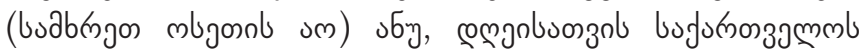

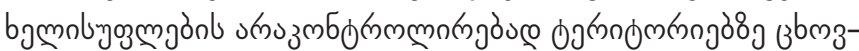

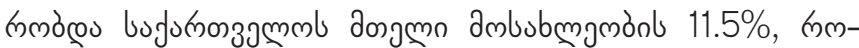

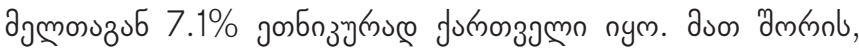

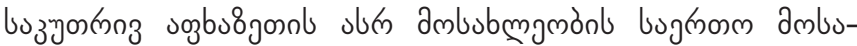

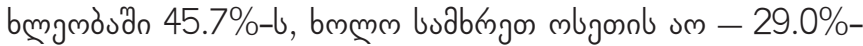

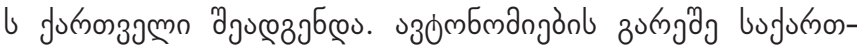

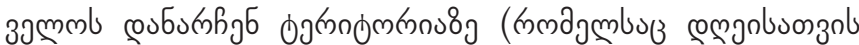

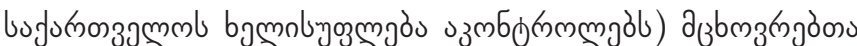

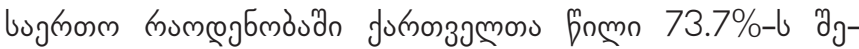

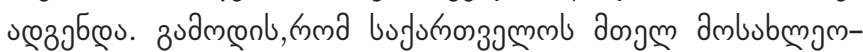

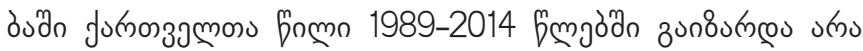

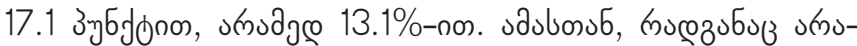

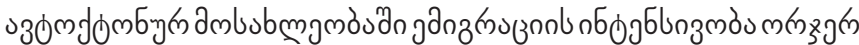

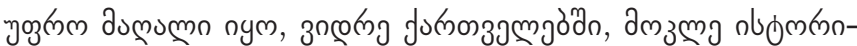

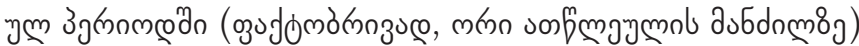

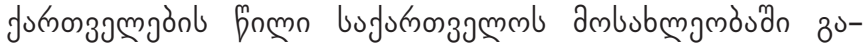

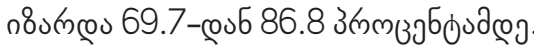

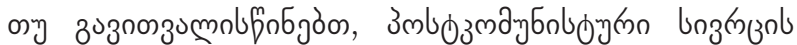

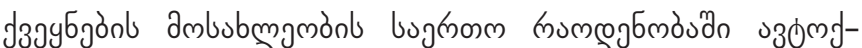

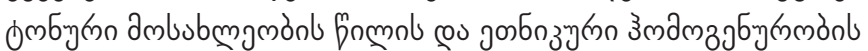

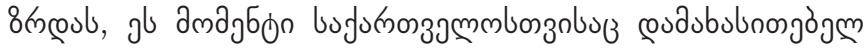

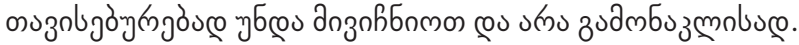

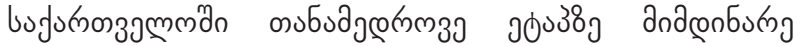

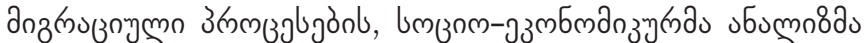

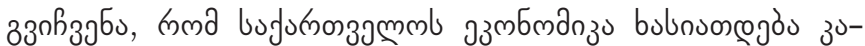

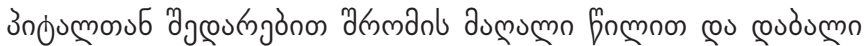

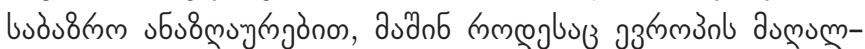

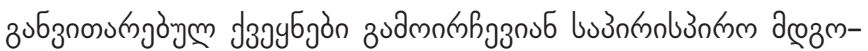

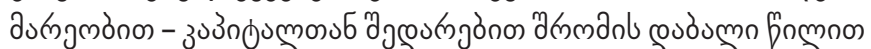

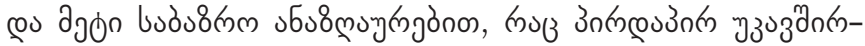

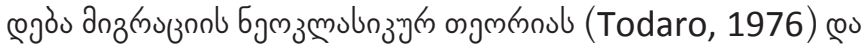

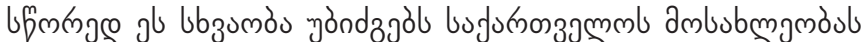

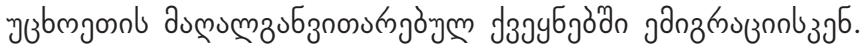

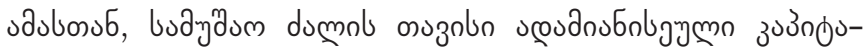

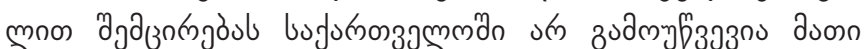

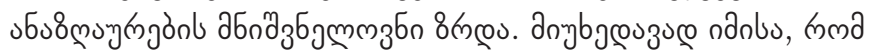

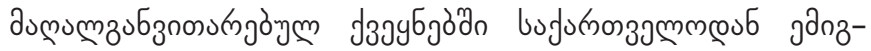

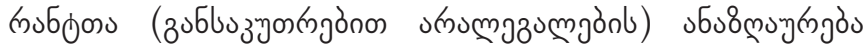

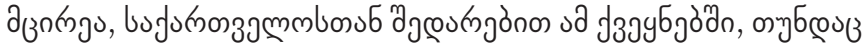

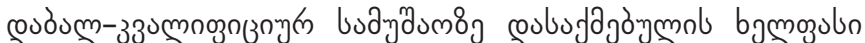

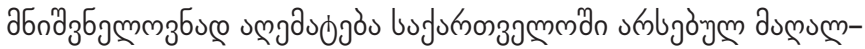

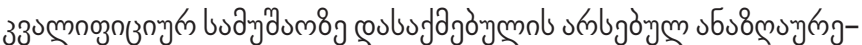

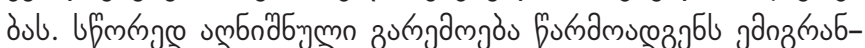

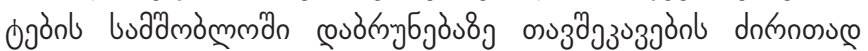
ano $88 \mathrm{sl}$

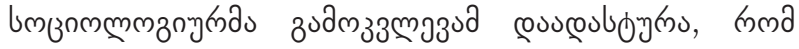

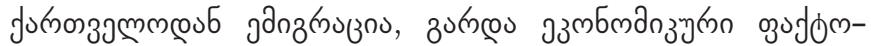

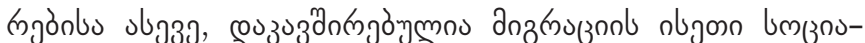

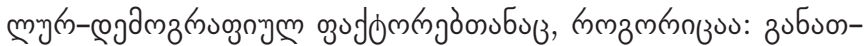

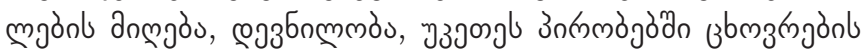

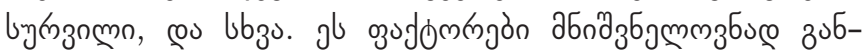

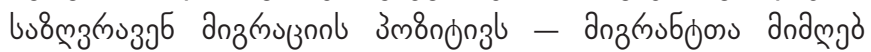

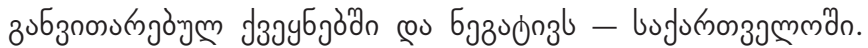

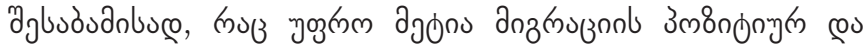

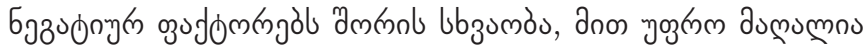

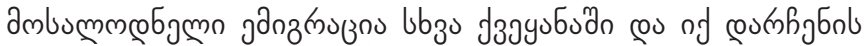

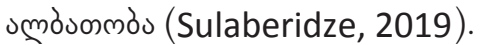

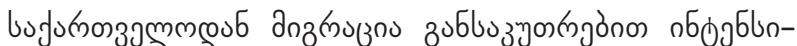

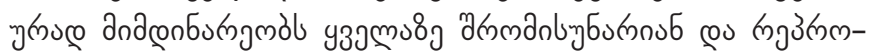

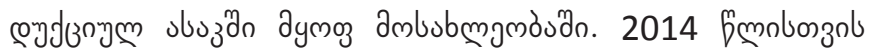

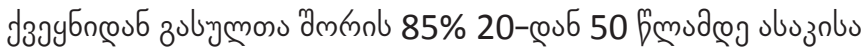

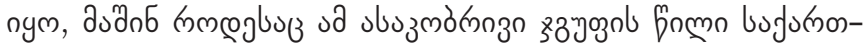

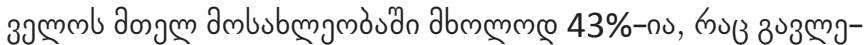

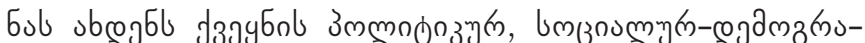

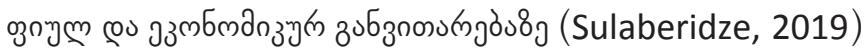




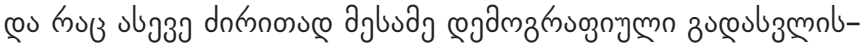
ozolus posabbulnuongòmm.

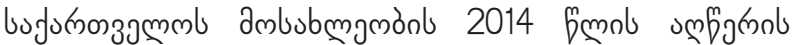

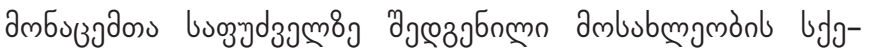

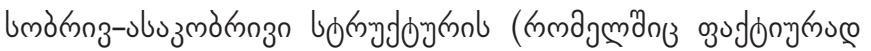

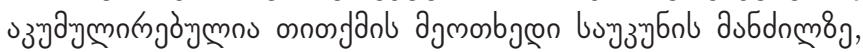

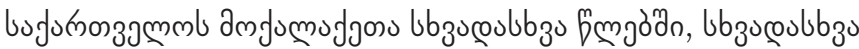

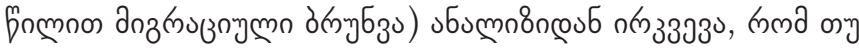

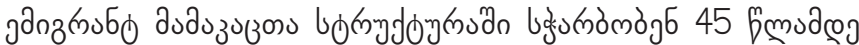

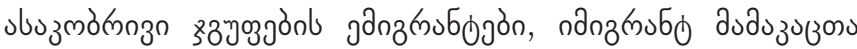

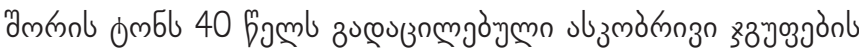

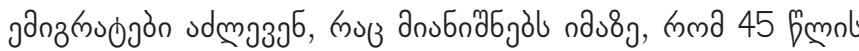

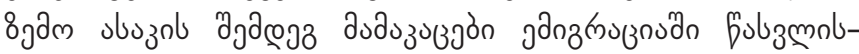

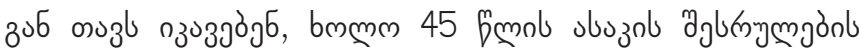

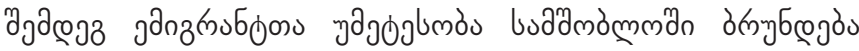
(Sulaberidze, 2018:95).

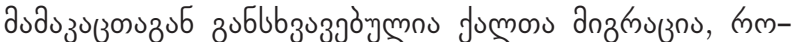

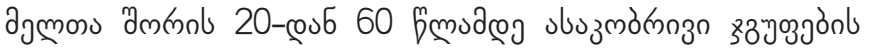

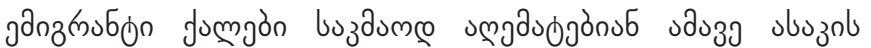

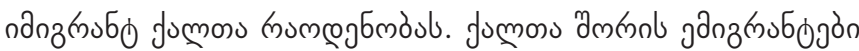

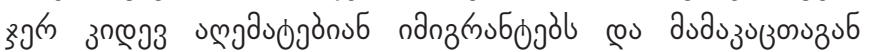

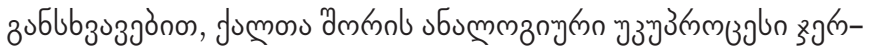

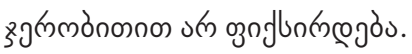

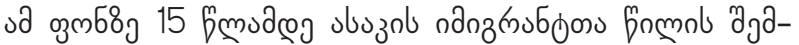

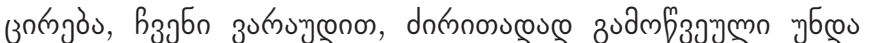

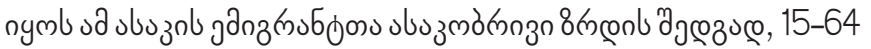

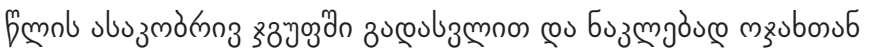

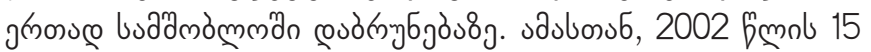

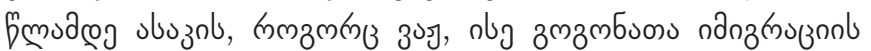
dugumn du

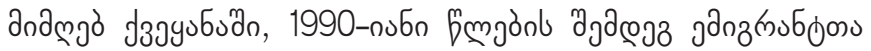

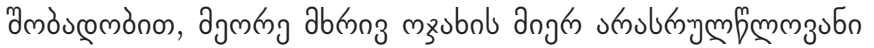

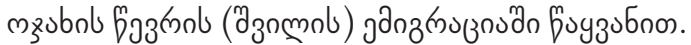

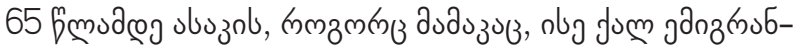

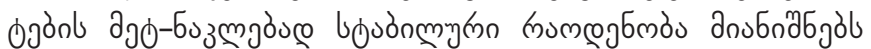

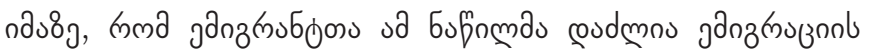

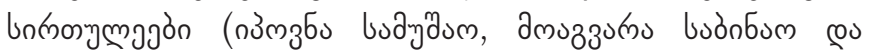

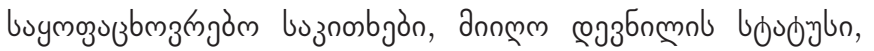

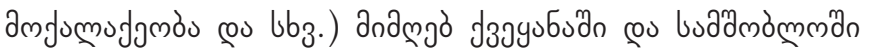

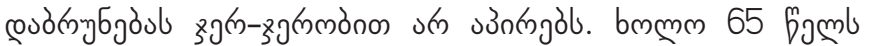

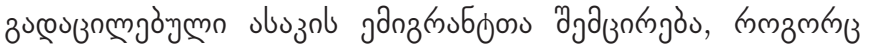

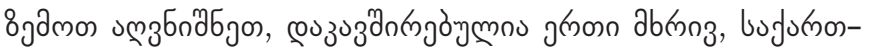

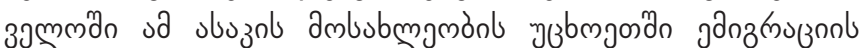

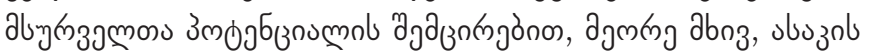

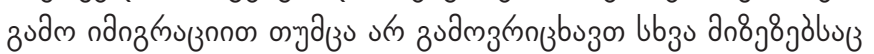

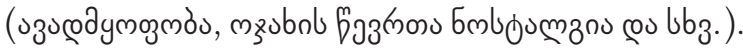

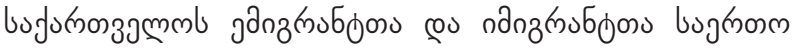

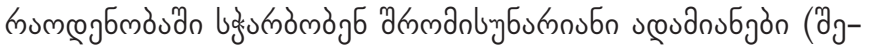

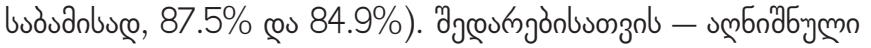

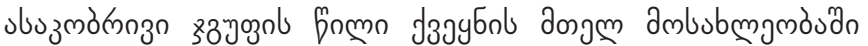

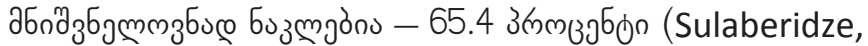
2018:97).

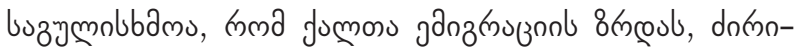

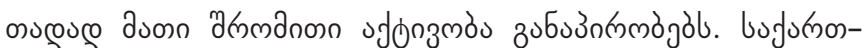

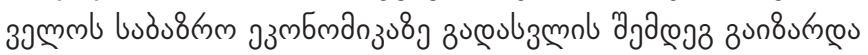

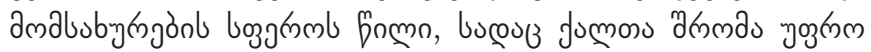

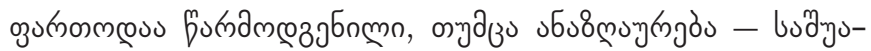

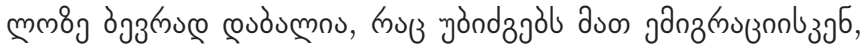

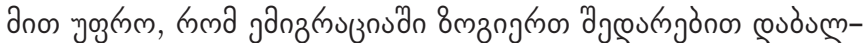

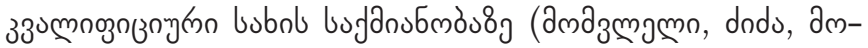

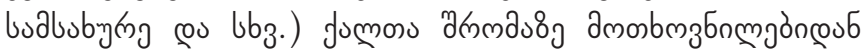

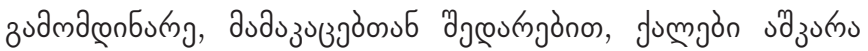

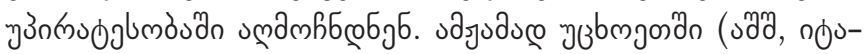

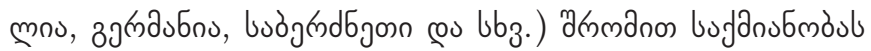

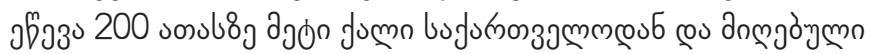

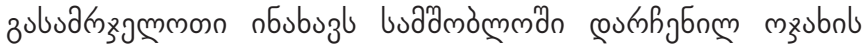
foз rigal (Shelia, 2018:138).

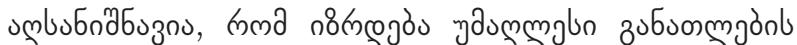

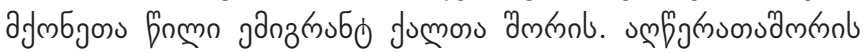

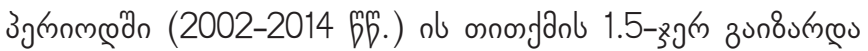

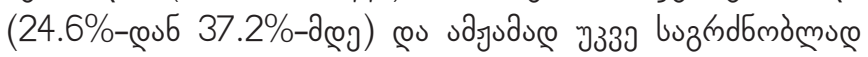

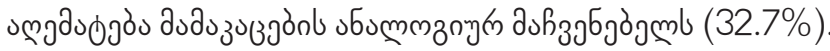

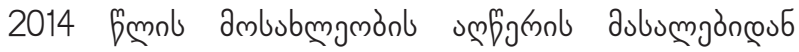

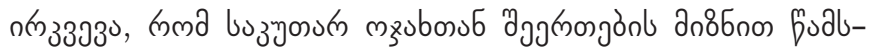

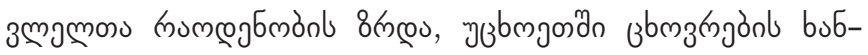

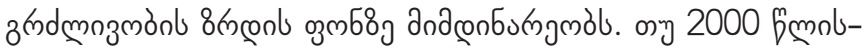

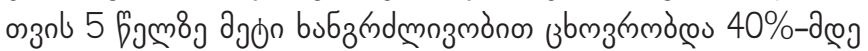

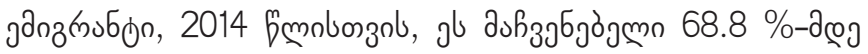

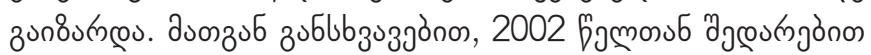

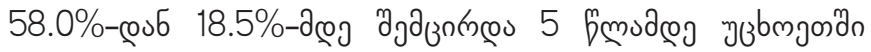

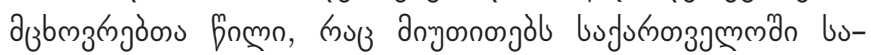

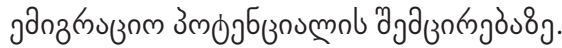

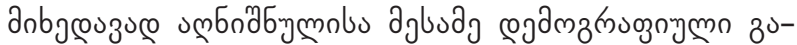

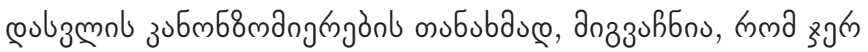

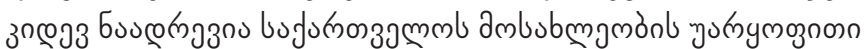

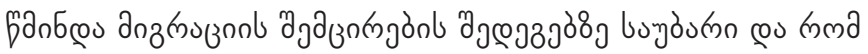

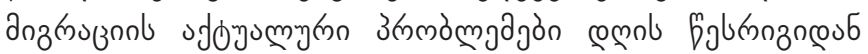

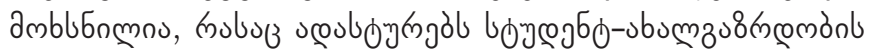

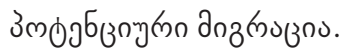

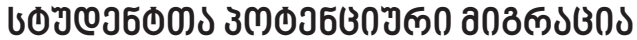

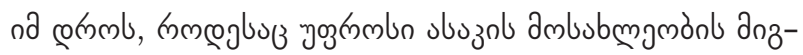

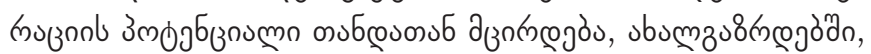

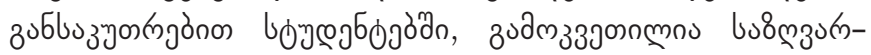

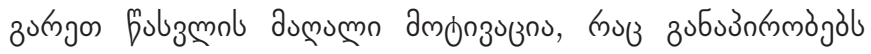

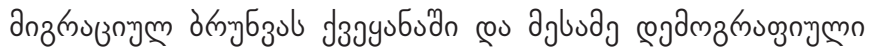

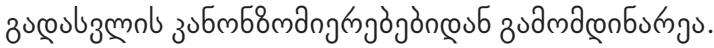

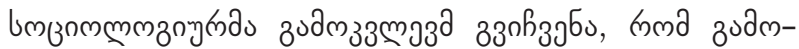




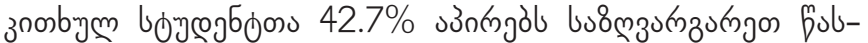

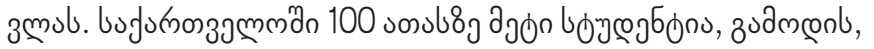

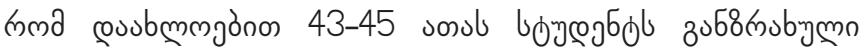

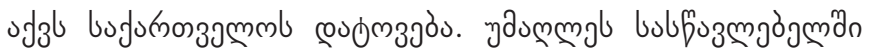

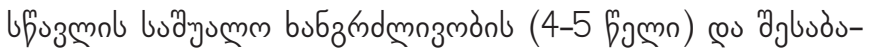

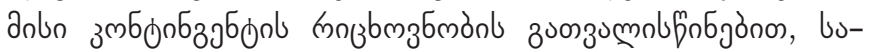

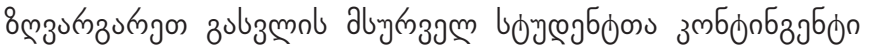

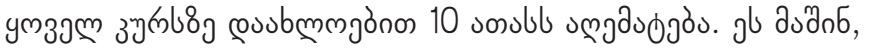

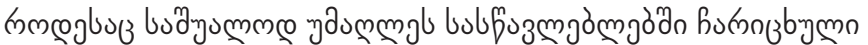

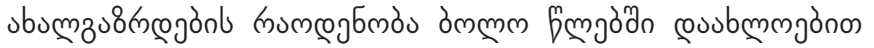
30-35 sonulob oुumzamgòno.

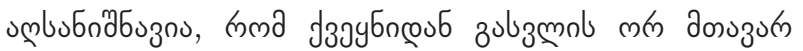

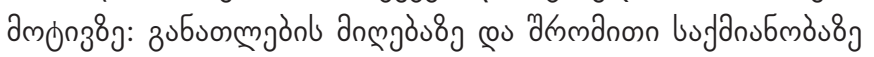

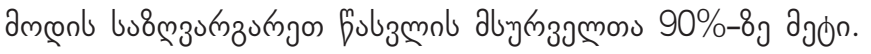

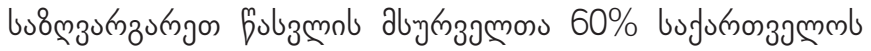

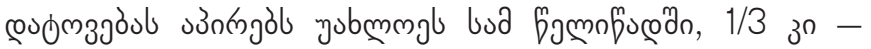

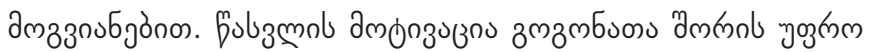

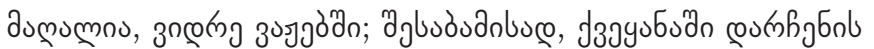

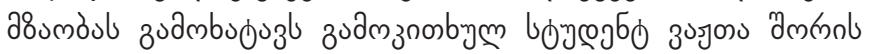

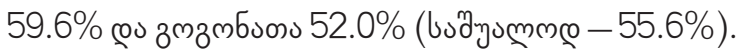

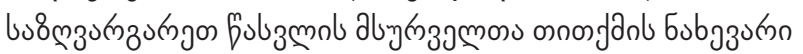

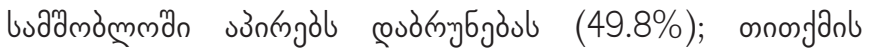

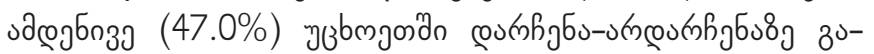

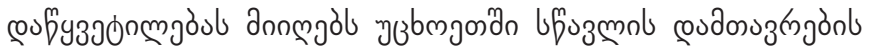

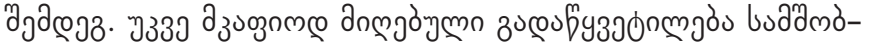

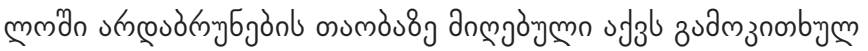

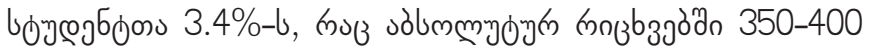
sbumzus

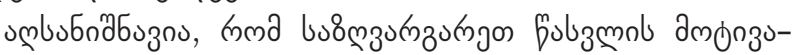

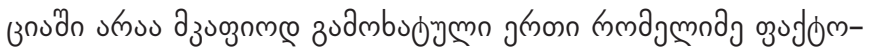

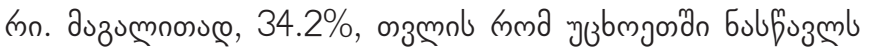

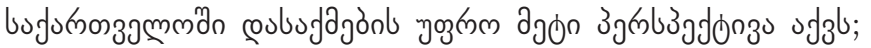

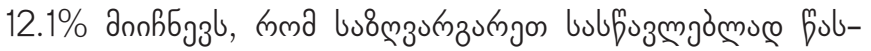

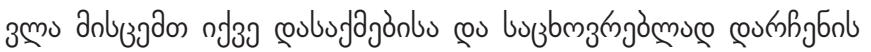

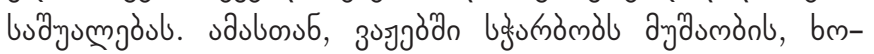
мm змзмб

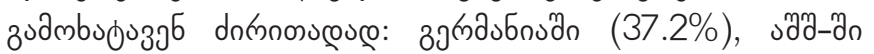

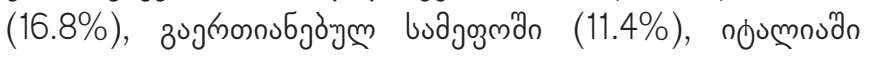
(5.4\%), buognubajoman pu ninbgoran (3.4-3.4\%).

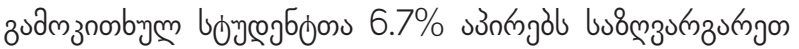

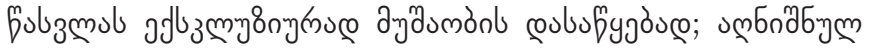

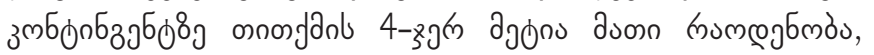

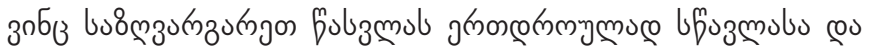

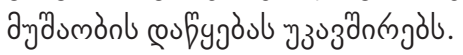

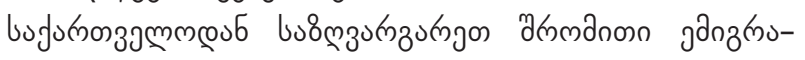

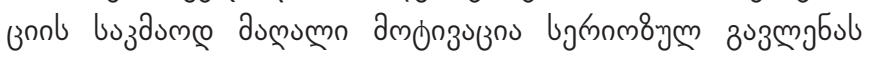

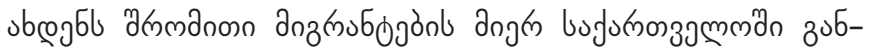

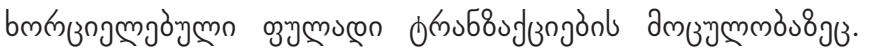

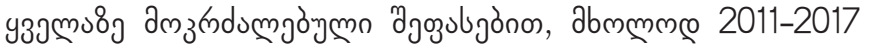

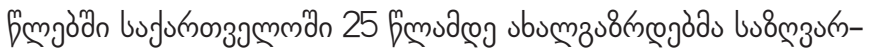

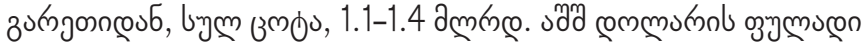

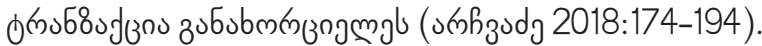

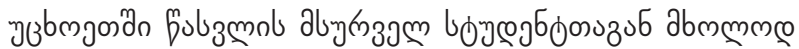

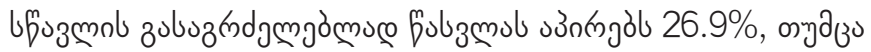

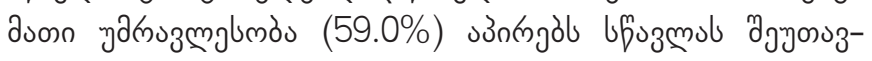

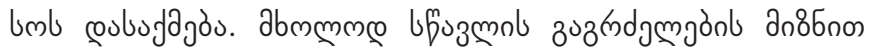

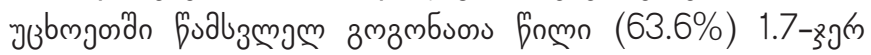

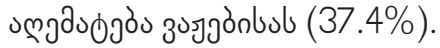

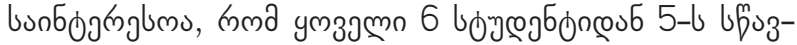

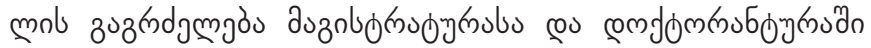

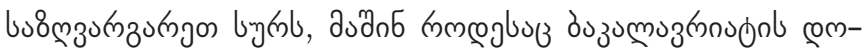

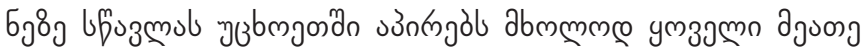
bojegbon.

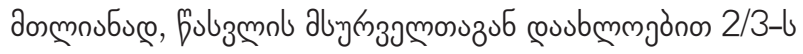

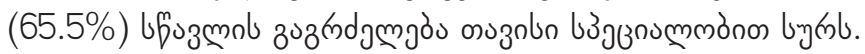

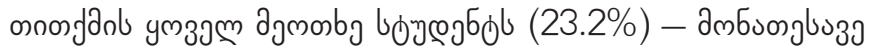

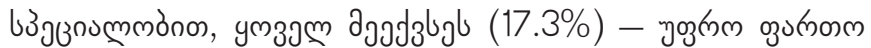

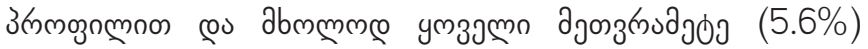

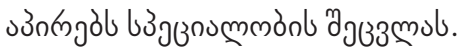

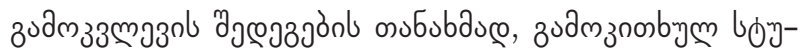

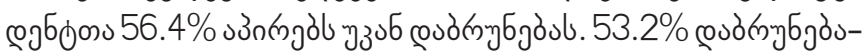

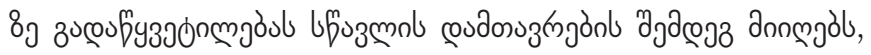

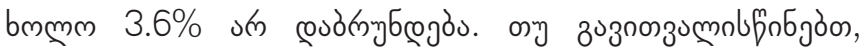

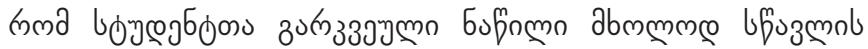

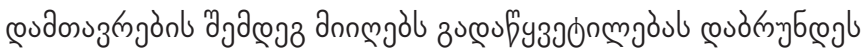

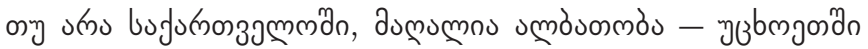

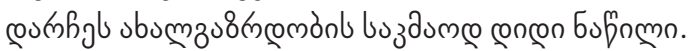

\section{cos335s}

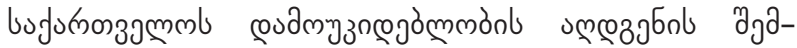

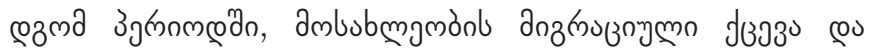

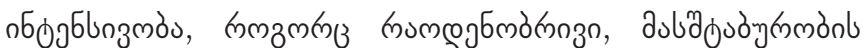

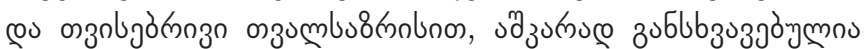

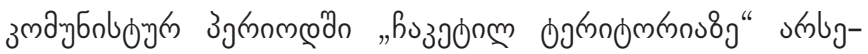

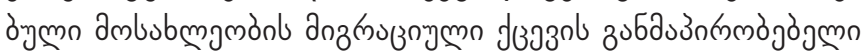

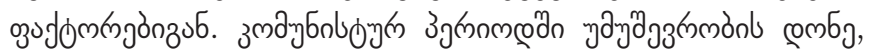

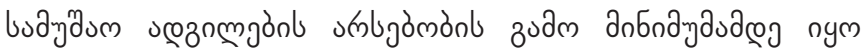

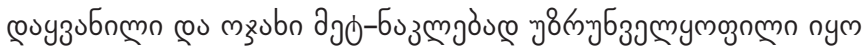

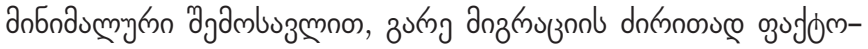

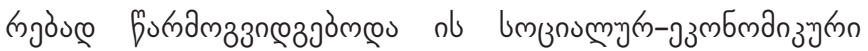

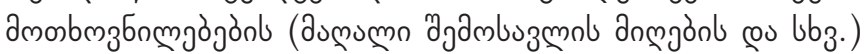

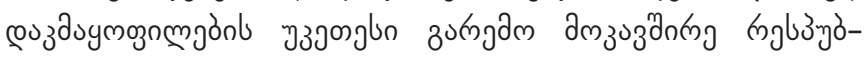

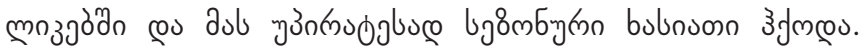

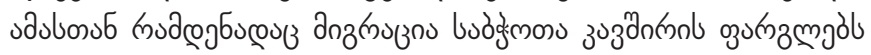

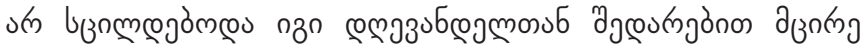
dubajuobyron nym.

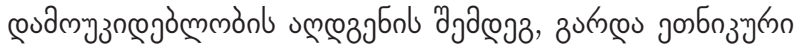

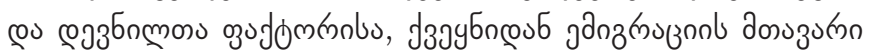




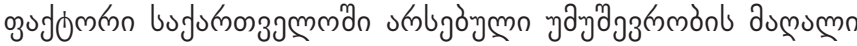

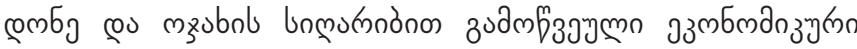

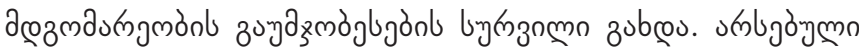

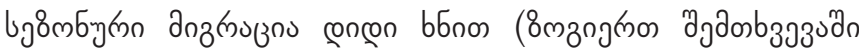

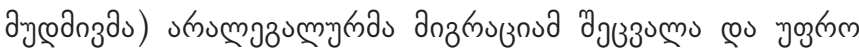

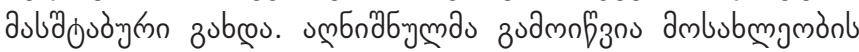

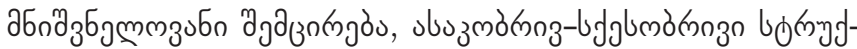

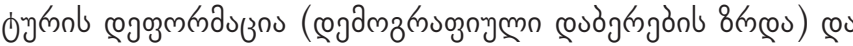

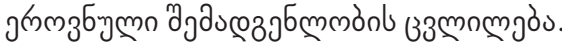

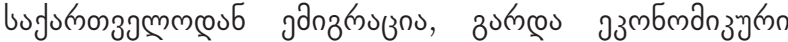

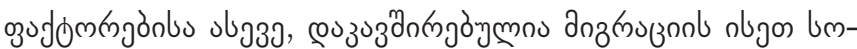

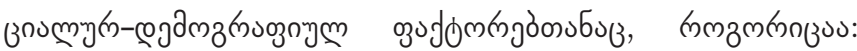
зง

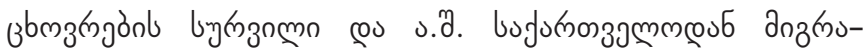

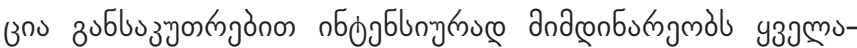

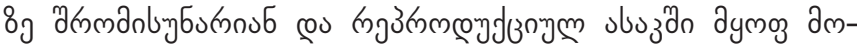

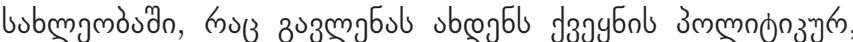

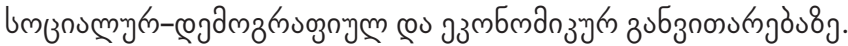

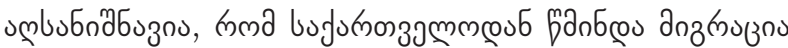

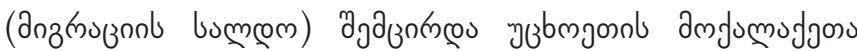

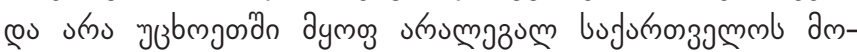

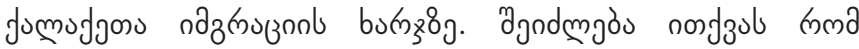

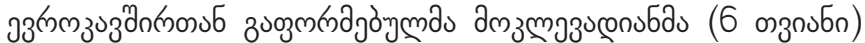

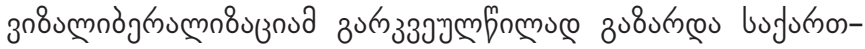

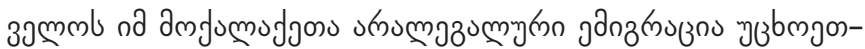

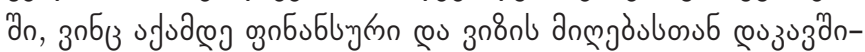

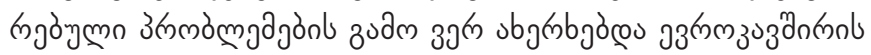

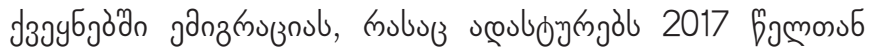

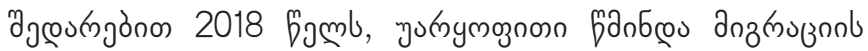

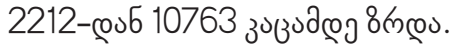

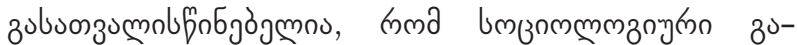

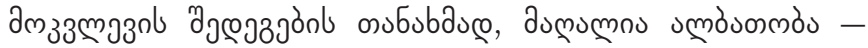

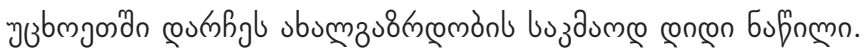

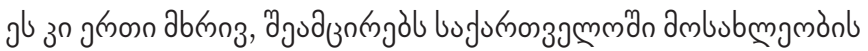

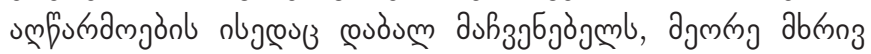

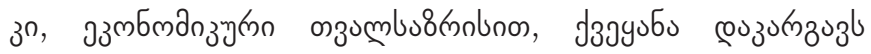

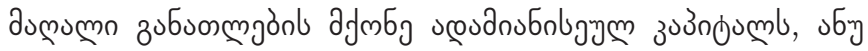

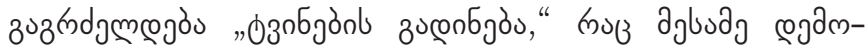

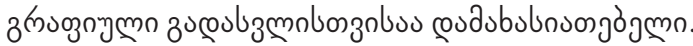

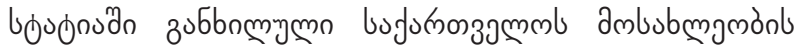

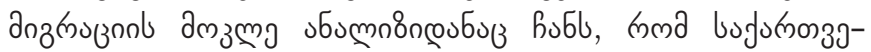

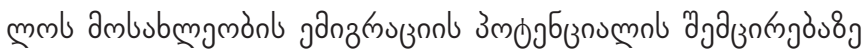

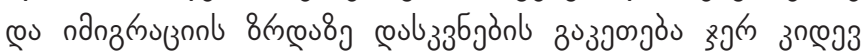
busconganos.

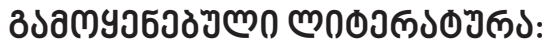

1. Archvadze J. (2018).On scales and repatriation of funds by labor migrants. Collection of works: urgent problems of migration of Georgian population [Shromiti migrantebis mier tankhebis repatriatsiisa da masshtabebis shesakheb]. Tbilisi, pp. 174-194. (In Georgian).

2. Sulaberidze A., Gvritishvili Ts. (2018). Third demographic transition [Mesame migraciuli gadasvla]. Collective monography: "Urgent problems of migration of Georgian population". Tbilisi, p. 23. (In Georgian).

3. Sulaberidze A. (2018). Historical periodization of the migratory processes of Georgian population [SaqarTvelos mosakhleobis migratsiuli protsesebis istoriuli periodizatsia]. Collective monography: "Urgent problems of migration of Georgian population". Tbilisi, p. 95. (In Georgian).

4. Shelia M. (2018). Peculiarities of women's labor emigration from Georgian [Saqartvelodan qalta shromiti emigratsiis taviseburebebi]. Collection of works: "Urgent problems of migration of Georgian population”. Tbilisi, p. 123. (In Georgian).

5. Materials of 2017 sociological research of the Institute of demography and sociology llia State University [Ilias sakhekmtsifo universitetis demografiis da sotsiologiis institutis 2017 tslis sotsiologiuri gamokvlevis masalebi].

6. Coleman D. (2004). Migration in the 21st Century. A Third Demographic Transition in the Making? Plenary Address to the British Society for Population Studies Ann. Conf. Leicester, 13 Sept.

7. Coleman D. (2006). Immigration and Ethnic Change in Low-Fertility Countries; A Third Demographic Transition (Statistical data) \\Population and Development Rev. Sept. Vol. 32. №3.

8. Hakkert, R. (2017). Population Dynamics in Georgia. An Overview Based on the 2014 General Population Census Data. pp. 60-69.

9. International Migration. 2013. ST/ESA/ SERA/338. N.Y.: United Nations

10. Landry A. (1986). On the Demographic revolution // Population and development Review. Vol. 13 (4). P. $731-740$.

11. Van De Kaa D. (1976). Login-term population policies in Europe // Seminar on the implications of a stationary or declining population in Europe, September 6-10, Strasburg, 1976. Strasburg: Council of Europe, 45 p.

12. Zelinsky Z. (1971). The Hypothesis of the Mobility Transition. Geographical. Review 61(2).

13. Vishnevski A. Time of demographic change [Vremia demograficheskix peremen]. M., 2015: 319. (In Russian).

14. Sulaberidze A., Archvadze J., Sualberidze V. Major factors and structure of migrants in post-communist Georgia [Osnovnie faktori I struqtura migrantov v post-kommunisticheskoi Gruzii] (published in the journal "Demographic Review. A perreviewed open-accses electronic journal" (In Russian). https://demreview.hse.ru/

15. www.un.org 


\title{
MIGRATION OF GEORGIAN POPULATION AGAINST THE BACKGROUND OF THE THIRD DEMOGRAPHIC TRANSITION
}

\author{
AVTANDIL SULABERIDZE \\ Doctor of Economic Sciences, professor, \\ Ilia State University, \\ Institute of Demography and Sociology, \\ Academtion of Academy of Economic Sciences of Georgia, Georgia \\ sulaberidzeavtandil@gmail.com
}

KEYWORDS: EMIGRATION, IMMIGRATION, THIRD DEMOGRAPHIC TRANSITION, POTENTIAL MIGRATION OF STUDENTS, VISA LIBERALIZATION.

For citation: Sulaberidze, A. (2019). Migration of Georgian population against the background of the third demographic transition, Globalization And Business, №8, pp. 96-104. https://doi.org/10.35945/gb.2019.08.011

\section{SUMMARY}

An important role of migration in the world as well as in local predictable changes, with regard to certain countries' population, indicates to the challenge of migratory processes. In order to explain the causes of migration, with the aim of its optimization and regulation, there were formulated various theories and concepts regarding the migration. From among these concepts, we have employed the neoclassical theory of migration and the theory of migratory transition, as well as the concept of the third demographic transition.

In the process of the formation of Georgian population, migration played, and still does, a significant part in influencing the growth rate of the Georgian population's size. If natural increase was the main component in terms of the size formation of the Georgian population during 1960-1991, from 1992 up until today, external migration assumed the same role. Since 1992, the low natural increase can no longer compensate for the negative net migration as a consequence of which the Georgian population has decreased by 3723.5 thousand persons by 2019.

On the one hand, since 1992, Georgia has belonged to the list of the countries that are demographically developed and on the verge of depopulation. On the other hand, because of the high intensity of emigration, it is among the donor countries which supply the developed countries with emigrants. Socio-economic analysis of the migratory processes that are associated with Georgian population has showed us that Georgia's economy is characterized by a high share of labor in comparison to capital and by low market income, whereas the highly-developed countries of Europe are distinguished by the opposite tendency - the low share of labor compared to the capital and higher market income.
This is directly linked to the neoclassical theory of migration, and the mentioned distinction forces Georgian population to emigrate toward the highly-developed foreign countries. Furthermore, decreasing of work-force together with its human capital did not cause a significant growth of their income. In spite of the fact that the salary of the Georgian emigrants (especially illegal ones) is small in the highlydeveloped countries, in comparison to Georgia, European countries offer substantially higher salaries even on the low-qualification jobs than the actual salaries of a worker employed on the high-qualification jobs in Georgia will ever be. This difference is the main reason as to why the emigrants refrain from returning to their homeland.

A sociological survey has confirmed that emigration from Georgia, besides economic factors, is associated with such social-demographic factors of migration as are: receiving education, exile, and the desire to live in better conditions, etc. These factors substantially determine the positive of migration in the receiving highly-developed countries and negative - in Georgia. Therefore, the more distinction there is between the positive and negative factors of migration, the higher the expected emigration to the other countries and the possibility of staying there. According to the current statistical information with regard to the materials of the 2014 population census, the article demonstrates the dynamics and tendency of the migration of the Georgian population since the country gained its independence up until today. It analyzes migrants' sex-age structure. It has been revealed that the emigration is especially intensive amid the population that is in their reproductive age and is ablebodied. Moreover, it is related to social-economic factors.

The sociological research conducted on students showed us that $42 \%$ of them intend to emigrate abroad 
after they graduate during 2018-2020. Additionally, working is the primary motive for boys as studying is for girls. After receiving education abroad, the half of them intend to return to homeland provided they can find a job with a decent salary in Georgia.

Net migration has decreased at the expense of the immigration of foreign citizens and not at the cost of illegal Georgian citizens. In the aftermath of a short-term visaliberalization within the territory of the $\mathrm{EU}$, emigration from
Georgia has increased, which is confirmed by the growth of the negative net migration from 2212 to 10763 inhabitants in 2017-2018. This, in turn, will reduce the population reproduction potential in Georgia and through "brain drain" the country will lose its human capital that possesses higher education.

Therefore, it is still early to draw conclusions with regard to improving the perspectives of emigration and immigration of Georgian population. 